\title{
Daseinsvorsorge in Gefahr: öffentliche Bibliotheken zwischen Digitalisierung und Austerität
}

\author{
Katja Thiele \\ Department of Geography, University of Bonn, Bonn, Germany \\ Correspondence: Katja Thiele (kthiele@uni-bonn.de)
}

Received: 2 October 2019 - Revised: 3 March 2020 - Accepted: 28 April 2020 - Published: 20 May 2020

\begin{abstract}
Kurzfassung. Public libraries are an important public service of general interest and, as part of social infrastructures, contribute to educational justice. This article discusses their development against the background of the interaction of digitization and austerity. As voluntary services they are particularly affected by the current austerity policy and have to increasingly justify their services and structures. At the same time, they are being rediscovered in the course of local authorities' urban development strategies. Based on empirical results from Bonn, the paper discusses central municipal strategies for the further development of the local library system and sheds light on the associated ambivalent socio-spatial implications.
\end{abstract}

\section{Einleitung}

Bibliotheken werden oft unterschätzt. Was passiert da schon? Menschen lesen Bücher, leihen sie aus und geben sie ab. Das klingt verstaubt und nicht modern. Doch die Bibliothek gehört noch immer zu den beliebtesten Orten der Freizeitgestaltung und das Lesen von Zeitungen, Zeitschriften und Büchern rangiert in Deutschland unter den 15 häufigsten Freizeitbeschäftigungen von Erwachsenen (hbz, 2016). Öffentliche Bibliotheken richten sich an Menschen allen Alters, insbesondere aber an nachwachsende Generationen: von ca. 400000 Veranstaltungen, die jährlich von oder in öffentlichen Bibliotheken durchgeführt werden, hatten 2017 beachtliche $44,8 \%$ Kinder und Jugendliche als Zielgruppe (dbv e.V., 2019). Öffentliche Bibliotheken sind somit nicht nur Bestandteil alltäglicher sozialer Infrastrukturen, sondern ergänzen vorhandene Einrichtungen kommunaler Bildungslandschaften (Duveneck und Volkholz, 2011) und sind ein wichtiges Element kommunaler Daseinsvorsorge mit besonderer Bedeutung für Bildungsgerechtigkeit und gesellschaftliche Teilhabe. In Mittel- und Kleinstädten sind sie oft eine zentrale oder sogar die einzige außerschulische Bildungseinrichtung und ,,[u]m [...] in ländlichen Regionen [solche] Kulturangebote zu nutzen, müssen [... Nutzer*innen lange Wege in die nächst größere Mittel- oder Großstadt zurücklegen“, denn trotz ihrer Bedeutung leisteten sich 2018 nur
3900 der 11254 deutschen Gemeinden eine kommunale öffentliche Bibliothek (exkl. Zweigstellen) (Seefeldt, 2018).

Obwohl öffentlichen Bibliotheken diverse Rollen in der städtischen Bildungslandschaft zukommen, sind sie bisher kein Thema der geographischen Stadtforschung. Vor dem Hintergrund der Veränderung der Wahrnehmung und Funktionen von Bibliotheken beschäftigt sich dieser Beitrag mit der Frage, wie sich öffentliche Bibliotheken im Spannungsfeld von Digitalisierung und Austerität entwickeln und diskutiert die These, die Digitalisierungsstrategie in Bibliotheken verbessere das Angebot und den Zugang für die breite Stadtbevölkerung.

Bereits seit Ende der 1990er Jahre „orientieren sich öffentliche Bibliotheken [...] zunehmend am Leitbild eines modernen Kommunikations- und Informationszentrums“ (Freytag und Hoyler, 2002:102), denn in den letzten drei Dekaden wurde das analoge Buch als dominantes Unterhaltungsmedium abgelöst durch eine wachsende Diversität an digitalen Medien sowie Apps und Online-Tools. Dies lässt den $\mathrm{Zu}-$ gang zu Medien und Wissen einfacher denn je erscheinen und stellt öffentliche Bibliotheken als physische Orte in Frage. Sowohl Wissenschaftler*innen als auch Praktiker*innen haben Bibliotheken als Abhol-Ort analoger Medien deshalb in der jüngeren Vergangenheit immer wieder ihren nahenden Niedergang prognostiziert (Knoche, 2018:11ff.). Öffentlichen Bibliotheken wurde dementsprechend lange Zeit wenig 
Aufmerksamkeit seitens der Verantwortlichen in Stadtentwicklung und -planung geschenkt und sie wurden, wie auch öffentliche Schwimmbäder, Schulen und weitere Einrichtungen der Daseinsvorsorge, im Zuge einer ,andauernden Krise öffentlicher Haushalte" (Silomon-Pflug und Heeg, 2013:184) regelrecht vernachlässigt (Mattert et al., 2017:36ff.). Als freiwillige kommunale Leistung sinkt zudem seit den 1990er Jahren durch Finanzknappheit die Anzahl der Bibliotheken auf Bundesebene (Freytag und Hoyler, 2002:102). In den daraus resultierenden Wandlungsprozessen haben sich in den letzten Jahrzehnten auch die relevanten Akteur*innen, ihre Interessen und Governance-Strukturen sowie ihre sozialräumlichen Strukturen verändert, wie im empirischen Teil am Beispiel Bonns illustriert wird.

Dem Narrativ des Bibliothekssterbens gegenüber steht allerdings auch eine Erzählung, die Bibliotheken als moderne dritte Orte ${ }^{1}$ imaginiert (Aabø und Audunson, 2012). Sie seien auch in Zeiten der Digitalisierung Begegnungsorte, Orte der Freizeitgestaltung und des (lebenslangen) Lernens sowie ein „Garant für die Attraktivität der [Innen]Stadt“ (Roeder, 2018). Als Teil kommunaler Digitalisierungsstrategien wird ihnen nicht selten eine bedeutende Rolle in einer modernen Stadtentwicklung zugesprochen und auch Bibliotheksleitungen und -mitarbeiter*innen bemühen sich um eine stärkere (multimediale) Öffentlichkeitsarbeit und die Zusammenarbeit mit Stadtplaner*innen, um ihre Potentiale sichtbarer zu machen. Paradoxerweise scheint also gerade die Digitalisierung die Perspektiven der Bibliothek positiv zu verändern und sie ins Rampenlicht von Stadtentwicklungsprozessen zu rücken. Deutlich wird das nicht zuletzt an zahlreichen Bibliotheksneubauten, die in den letzten 10 Jahren als Leuchtturmprojekte entstanden (Abschnitt 3.2) und Teil von umfassenden Stadterneuerungsplänen sind.

Im Folgenden wird darauf eingegangen, welche gesellschaftlichen Funktionen öffentlichen Bibliotheken zukommen und diese werden in Beziehung gesetzt zu den damit verbundenen Raumverständnissen (Abschnitt 2). AnschlieBend wird dargestellt, wie öffentliche Bibliotheken im Zuge einer kommunalen Finanzknappheit unter Druck geraten, mit welchen Strategien Kommunen darauf reagieren und welche Rolle Digitalisierungsprozesse dabei spielen (Abschnitt 3). Die Veränderungen der Angebotsstruktur im kommunalen Bibliotheksnetz lassen sich besonders deutlich am Beispiel von Großstädten darstellen, da diese mehr als einen Bibliotheksstandort unterhalten und ihr gesamtes Bibliothekssystem neu organisieren müssen. Am Beispiel empirischer Er-

\footnotetext{
${ }^{1}$ Der Begriff des dritten Ortes wird im Zusammenhang mit Bibliotheken von Akteur*innen auf allen Ebenen sowie Wissenschaftler*innen aufgegriffen und verweist auf eine längere Diskursgeschichte, die an dieser Stelle nicht wiedergegeben werden kann. Ursprünglich geht er zurück auf die Konzeptionen von third space und thirdspace von Bhabha (1994) und Soja (2007). Weiterentwicklungen des Begriffes von thirdspace im Sinne von place (also eines konkreten Ortes) bieten stadtsoziologische Überlegungen von Oldenburg (1989:20ff.).
}

hebungen in Bonn wird demonstriert, dass diese Reorganisation im Zuge von kommunalen Digitalisierungsstrategien jedoch ambivalent ist (Abschnitt 4). Die daraus resultierenden sozial-räumlichen Implikationen werden im Fazit zusammenfassend betrachtet.

\section{Funktionen öffentlicher Bibliotheken}

Öffentliche Bibliotheken haben unterschiedliche gesellschaftliche Funktionen, die sich nur teilweise in formalen Regelungen widerspiegeln. Mit der Frage, was Bibliotheken sind und welche Funktionen sie als Teil der öffentlichen Daseinsvorsorge erfüllen können, beschäftigen sich insbesondere die Informations- und Bibliothekswissenschaften (Gradmann und Umlauf, 2012:25). Die Funktionen von öffentlichen Bibliotheken stehen in einem engen Verhältnis zu den Verständnissen von Raum, sowohl von der Bibliothek an sich als auch von ihrer Rolle für die Stadt (Abb. 1).

Öffentliche Bibliotheken werden schon immer als Orte des Aufbewahrens und des Zugangs zu Medien verstanden. Sie waren und sind damit zugleich „Systeme für die Wissensrepräsentation und Wissensordnung" ihrer jeweiligen Zeit (Gradmann und Umlauf, 2012:4). Als Räume des Wissens und Lernens und als Gedächtnisinstitution bilden sie das kollektive kulturelle Gedächtnis (ab) und machen es zugänglich (Hobohm, 2017). Im Zuge der Digitalisierung wird der Zugang zu Medien jedoch neu geordnet und ausgeweitet: Neben das Abholen von Medien am physischen Ort treten virtuelle Zugänge auf digitalisierte Bücher, E-Books, elektronische Zeitschriften, Musik, Filme oder Spiele.

„Die Bibliotheks- und Informationswissenschaft versteht Bibliothek [traditionell] als nicht-kommerzielle Einrichtung“" (Gradmann und Umlauf, 2012:29) und die zur Verfügung stehenden Medien als „quasi-öffentliche Güter“ (Gradmann und Umlauf, 2012:30), die den öffentlichen Daseinsvorsorgeauftrag decken. Als Einrichtungen des außerschulischen Bildens und Lernens sind sie jedem Menschen zugänglich und durch ihre niedrigschwelligen Angebote (Audunson, 2005:434ff.) ermöglichen sie quer durch alle Bevölkerungsgruppen den gleichberechtigten Zugang zu Informationen, Wissen und sozialen Kontakten und tragen zur Verbesserung von Bildungschancen auch bildungsferner Menschen bei (Birdi et al., 2008:583; Di Marino und Lapintie, 2015:119; Gradmann und Umlauf, 2012:18f.). Im Wettbewerb um den Zugang zu Netzwerken, Jobs, Qualifikationen und damit verbundene soziale Aufstiegschancen erfüllen sie eine ganze Reihe wirtschaftlicher Funktionen; ihnen kommt aber auch eine wichtige Rolle für die Erhaltung und Stärkung von Demokratie und den Prinzipien wohlfahrtsstaatlicher Versorgung zu (DiMarino und Lapintie, 2015:119f.). Die Debatten in den Bibliotheks- und Informationswissenschaften sind hier direkt anschlussfähig an sozial- und raumwissenschaftliche Debatten zu öffentlichen Räumen und Konzepten von Third Space 


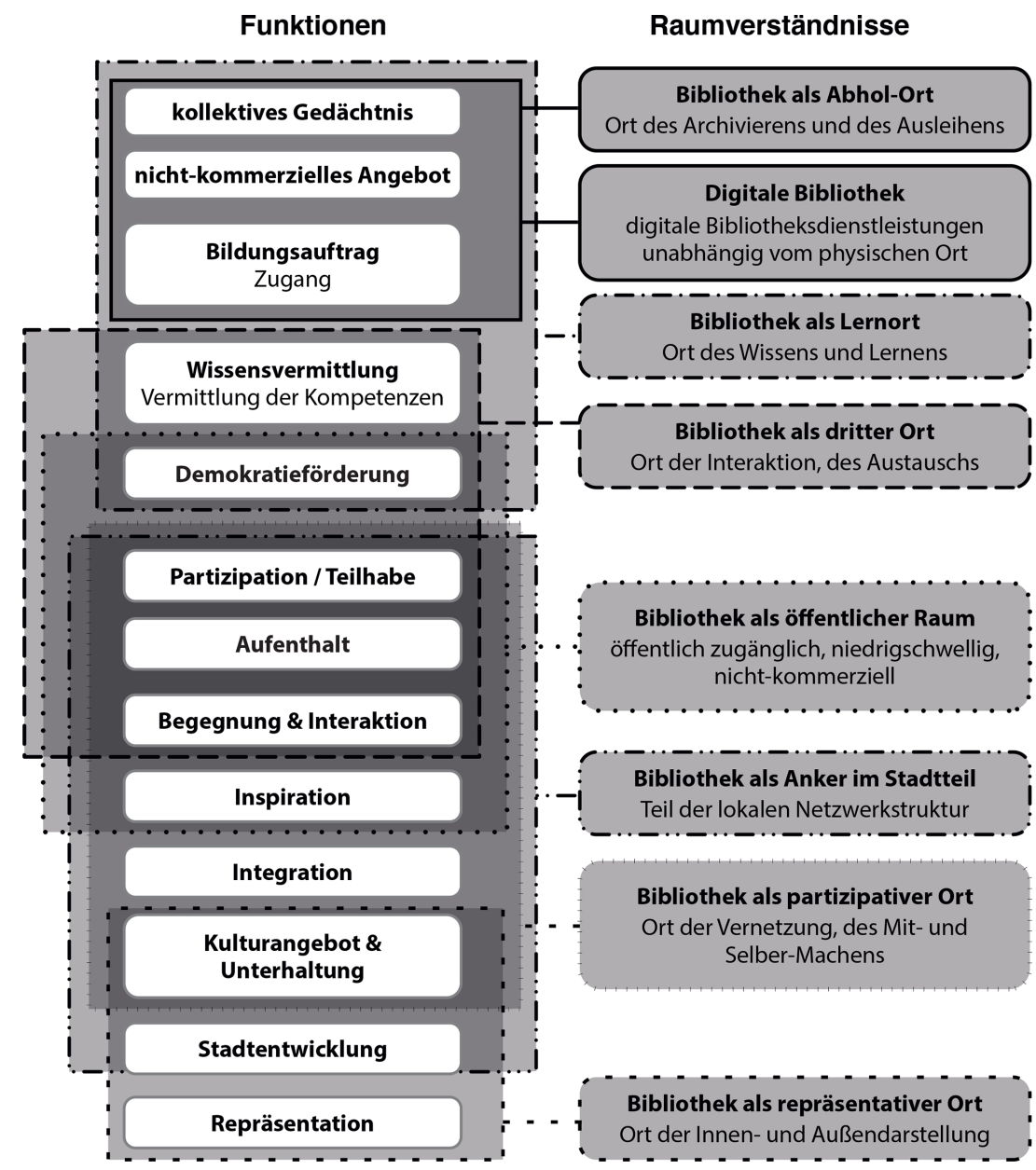

Abb. 1. Öffentliche Bibliotheken: Funktionen und Raumverständnisse. Grafik: Katja Thiele \& Irene Johannsen.

(Fußnote 1), die die Produktion und Relevanz öffentlicher Orte für gesellschaftliche Teilhabe betonen.

Öffentliche Bibliotheken sind lokal ,,verankert und vernetzt" (Freytag und Jahnke, 2015:83). Als Orte der Begegnung, der Interaktion und des Austauschs dienen sie Kindern, Jugendlichen und Erwachsenen als soziale und kommunikative Treffpunkte und Anker der sozialen Integration (Aab $\varnothing$ und Audunson, 2012:143). Die Funktionen öffentlicher Bibliotheken unterscheiden sich jedoch nach ihrer StadtgröBe. Während sie im Netzwerk kommunaler Bildungslandschaften größerer Städte nur ein Baustein sind, stellen sie in Mittel- und Kleinstädten sowie im ländlichen Raum oftmals eine zentrale oder die einzige außerschulische Bildungseinrichtung dar und müssen diverse Ansprüche und Funktionen erfüllen (Seefeldt, 2018). In Großstädten wie Bonn besteht das Bibliotheksnetz dagegen aus mehreren, sich ergänzenden Bibliotheksstandorten. Stadtteil- und Bezirksbibliotheken kommt die Aufgabe der Versorgung der Nutzer*innen mit einem Grundstock an Literatur und anderen Medien zu. Als zentrale soziale Infrastruktur des Alltags erreichen sie ihre Nutzer*innen im direkten Wohnumfeld. Zentralbiblio- theken bieten diese soziale Funktion ebenfalls für ihr direktes Umfeld, beherbergen darüber hinaus jedoch einen gröBeren Bestand und haben Repräsentationsfunktionen für die gesamte Stadt (nach innen und außen). Spezialbibliotheken bieten zusätzlich besondere Angebote in einem bestimmten Bereich (bspw. Musik).

Informations- und Bibliothekswissenschaftler*innen wie Birdi et al. (2008:579) zeigen, dass Orte der informellen Bildung besonders wichtige Beiträge zur Reduzierung sozialer Exklusion leisten ,for connecting vulnerable people to mainstream society". Angesichts wachsender sozial-räumlicher Disparitäten werden öffentliche Bibliotheken deshalb von Kommunen auch als potentiell wirkungsvolles Instrument der Stadtentwicklung zur Förderung von Integration, Inklusion, zivilgesellschaftlicher Partizipation sowie lokaler Strukturen der Mitbestimmung betrachtet, das den Zugang zu nationalen und kommunalen Bildungslandschaften eröffnet (Audunson, 2005). Für die Ausgestaltung dieses Auftrages ist jedoch der ,politische Wille [entscheidend, das Feld] [...] nicht allein den Märkten zu überlassen" (Gradmann und Umlauf, 2012:20), denn, welche Funktionen und Raumverständ- 
nisse gefördert werden, ist Teil der politischen Aushandlung auf kommunaler Ebene. Hier deutet sich bereits die Frage an, inwiefern die zunehmende Marktorientierung und die Forderung nach betriebswirtschaftlicher Effizienz im Widerspruch stehen zur Idee einer umfassenden wohlfahrtsstaatlichen Daseinsvorsorge und ihren sozialen Funktionen (Hobohm, 2013:624).

\section{3 Öffentliche Bibliotheken zwischen Austerität und Digitalisierung}

Neben den Funktionen öffentlicher Bibliotheken verändern sich auch ihre Angebote $^{2}$, ihre Organisation und die Zusammensetzung der an ihrer Entwicklung beteiligten Akteur*innen. Zur Veränderung der Angebotsstrukturen öffentlicher Bibliotheken tragen eine Reihe von Faktoren bei: Bibliotheken sind als zentrale Orte der Informationsgesellschaft maßgeblich von der wachsenden Bedeutung der $D i$ gitalisierung betroffen. Dort manifestiert sich wie Digitalisierung unser Leben auf allen Ebenen verändert - von der Einführung technologischer Innovationen und der Veränderung von Konsummustern, über die Reorganisation von Arbeitsprozessen, bis hin zur veränderten Kommunikation öffentlicher Einrichtungen mit ihrem Publikum. $\mathrm{Zu}$ den zentralen Treibern gehören, als Teil der bereits seit den 1980er Jahren beobachteten Neoliberalisierung und Internationalisierung von Städten (Peck, 2015), außerdem die knapper werdenden Ressourcen der öffentlichen Haushalte (insbesondere auf lokaler Ebene) sowie die damit verbundenen Effekte eines wohlfahrtsstaatlichen Wandels und der räumlichungleichen Entwicklung (Schipper und Schöning, 2016).

Neben diesen beiden Haupttreibern sind der demographische Wandel und der sozio-kulturelle Wandel als weitere Treiber von Veränderungen des Angebots zu nennen: Die vor allem in größeren Städten zunehmende Diversität der Bevölkerung (u.a. soziale Lage, demographische Merkmale, Migrationsbiographien, Lebensstile, Einstellungen) verändert zusammen mit Wachstums-, Schrumpfungs- und Alterungsprozessen die Nachfrage nach sozialen Leistungen und so die Anforderungen an die Angebotsseite (Winkel, 2006:173ff.; Libbe et al., 2010:17ff.).

Vor dem Hintergrund der vielerorts geführten Debatten um urbane Austerität (Schipper und Schöning, 2016) fokussiert dieser Artikel in erster Linie auf den ambivalenten Zusammenhang von Austerität (Abschnitt 3.1) und Digitalisierung (Abschnitt 3.2). Während Investitionen in öffentliche (soziale) Infrastrukturen in Deutschland in den letzten 15 Jahren drastisch abgenommen haben - dies gilt besonders für die kommunale Ebene, wo ein Großteil der Leistungen der Daseinsvorsorge erbracht wird (Mattert et al., 2017:19), werden

\footnotetext{
${ }^{2}$ Dieser Beitrag konzentriert sich auf die Angebotsseite öffentlicher Bibliotheken. Es sei jedoch darauf verwiesen, dass diese in einem engen Wechselverhältnis zu den Nutzer*innen und ihren Bedürfnissen (Nachfrage) steht.
}

öffentliche Bibliotheken im Zuge der Digitalisierung wiederentdeckt und stehen nicht selten im Zentrum kommunaler Stadtentwicklungsstrategien (Pollio, 2016).

\subsection{Freiwillige Leistungen gefährdet durch Austerität}

Die Bereitstellung von Infrastrukturen ist seit jeher eine politische Aufgabe der kommunalen Daseinsvorsorge, die entweder von der öffentlichen Hand selbst übernommen wird, durch entsprechende Investitionsanreize und Regulierungen gefördert oder an private Trägerstrukturen ausgelagert werden kann. Während Daseinsvorsorge einem öffentlichen Auftrag folgt, können Infrastrukturen sowohl privat als auch öffentlich finanziert und betrieben werden (Mattert et al., 2017). Im Hinblick auf Bildungsgerechtigkeit ist daher die Frage, wer für die Gewährleistung öffentlicher Infrastrukturen verantwortlich ist.

Seit den 1980er Jahren ist es zu einer Welle von Infrastruktur-Privatisierungen und effizienzorientierten Umstrukturierungen gekommen (Libbe et al., 2010; Wissen und Naumann, 2008). In der internationalen Literatur wird diese Entwicklung seit den 1990er Jahren unter dem Stichwort Neoliberalisierung und in jüngerer Zeit unter dem Begriff Austerität(spolitik) diskutiert (Brenner und Theodore, 2002; Peck, 2015; Schipper und Schöning, 2016:12). Seit den 2000er Jahren sind in Deutschland auch soziale Infrastruktureinrichtungen betroffen (Mattert et al., 2017:47). Der Bedeutung einer ergebnisorientierten, öffentlich finanzierten Daseinsvorsorge stehen in der Folge die sinkende staatliche Investitionstätigkeit in öffentliche Infrastrukturen (Mattert et al., 2017:36ff.) sowie die Fokussierung auf profitable Geschäftsfelder (Lebuhn, 2010:36f.) gegenüber.

Kommunen befinden sich am ,receiving end of austerity politics“ (Peck, 2012:634). Zu den Auswirkungen von Sparmaßnahmen auf die lokale Versorgungssituation und den Folgen für die Gleichwertigkeit der Lebensverhältnisse hat sich in der jüngeren Stadtforschung deshalb eine lebhafte Debatte unter dem Stichwort „Austerity Urbanism“ entwickelt (Peck, 2015). Öffentliche Bibliotheken sind Einrichtungen der öffentlichen Hand und liegen nach dem Recht auf kommunale Selbstverwaltung (Art. 28, Abs. 2, GG) in der Verantwortung der Kommunen. Ihre Unterhaltung gehört jedoch nicht zu den Pflichtaufgaben, sondern zu den freiwilligen Aufgaben von Kommunen. Da ihre Gewährleistung rechtlich und finanziell nicht abgesichert ist (dbv e.V., 2018:10), sind diese von Finanzknappheit besonders betroffen. Schon Anfang des Jahrtausends hatten nur ,etwa $90 \%$ der Kommunen mit mehr als 20.000 Einwohner[*inne]n“ und ,weniger als $50 \%$ der Gemeinden mit einer Einwohner[*innen]zahl [...] [unter] 20.000 eigene Bibliotheken“" (Freytag und Hoyler, 2002:102). Dass öffentliche Bibliotheken in Deutschland sich auch immer mehr „rechnen“ müssen, bestätigte bereits Hobohm (2007). Sie stehen in Konkurrenz zu anderen städtischen Einrichtungen der kommunalen Daseinsvorsorge und stärker unter Legitimations- und Kostendruck als Pflichtauf- 
gaben. An vielen Standorten sind sie mit einer Verringerung der ihnen zur Verfügung gestellten öffentlichen Finanzmittel und anderer Ressourcen konfrontiert, wie ein Blick in die Deutsche Bibliotheksstatistik ${ }^{3}$ zeigt. Mittlerweile können je nach Stadtgröße - $17 \%-25 \%$ aller öffentlichen Bibliotheken nur noch über einen reduzierten Medienetat verfügen (dbv e.V., 2016:11).

Als Reaktion auf den zunehmenden Finanzierungsdruck werden auf der kommunalen Ebene verschiedene Strategien angewandt, um den Betrieb von Bibliotheken effizienter und kostengünstiger zu gestalten (Abb. 2, eigene Empirie). Darunter fallen das Erschließen neuer Finanzquellen (durch Gebühren, Events, Medienverkäufe, Spendenboxen etc.), das Teilen von Aufgaben und Ressourcen (bspw. Verwaltung des Medienbestandes, Nutzung von Technik, räumliches Zusammenlegen verschiedener Einrichtungen) im eigenen Verbund oder über lokale Grenzen hinweg sowie die Trennung von Aufgaben oder das Outsourcen jener an Dritte (bspw. Verlagerung oder Auflösung von Beständen, Ausbau von Ehrenamtsstrukturen, Personaleinsparungen oder -abbau im Zuge von Open-Library-Modellen, Schließung von Stadtteilbibliotheken). Im Sinne der kostenorientierten öffentlichen Verwaltung (NPM) werden zudem auch im Bibliothekswesen neue Methoden der ökonomischen Leistungs- und Wirkungsmessung entwickelt und eingeführt (Gradmann und Umlauf, 2012).

Die kommunalen Bibliotheksstrategien lassen sich nicht scharf voneinander abgrenzen, da einzelne, auf den ersten Blick widersprüchliche, Maßnahmen parallel zum Einsatz kommen und ineinandergreifen (bspw. der Ausbau des Medienbestandes an einem und die Reduktion an einem anderen Standort). Es lässt sich jedoch eine Tendenz hin zu SharingModellen feststellen, die sowohl die räumliche Struktur als auch die interne Organisation, Personalausstattung und deren Finanzierung verändern.

Im Hinblick auf die Folgen dieser Veränderungen für die Bildungsgerechtigkeit ist besonders die räumliche $\mathrm{Di}$ mension interessant. Die „Abkehr von standardisierten und räumlich integrierten und eine Entwicklung hin zu stärker fragmentierten und räumlich ausdifferenzierten Infrastrukturen" (Graham und Marvin, 2008:38) wird unter dem Begriff „Splintering Urbanism“ seit einigen Jahren als schwer umkehrbare Folge der skizzierten Dynamiken thematisiert. ,[D]ie bisherige Auffassung von der ,Gleichwertigkeit der Lebensverhältnisse' als Gleichheit [werde schrittweise] zur Disposition gestellt“ (Barlösius, 2009:22). Die Debatte, wie und unter welchen Bedingungen von finanziellen Kürzungen betroffene bzw. teilweise privatisierte Infrastrukturen noch in der Lage sind, eine angemessene Versorgung der Bevöl-

\footnotetext{
${ }^{3}$ Die Deutsche Bibliotheksstatistik (DBS) wird vom Hochschulbibliothekszentrum des Landes Nordrhein-Westfalen (hbz) bereitgestellt und enthält alle wichtigen Kennzahlen öffentlicher und wissenschaftlicher Bibliotheken im Bundesgebiet Deutschland seit 1998.
}

kerung sicherzustellen, wird nicht nur in Deutschland kontrovers geführt. Auch McFarlane und Rutherford (2008:368) verweisen auf einen Zusammenhang von Finanzierungs- und Versorgungsproblemen bei Infrastrukturen und wachsender sozialer Ungleichheit. Obwohl Austerität häufig mit einem Versprechen auf mehr Gerechtigkeit einhergeht (Petzold und Duveneck, 2018:56), zeigen verschiedene Studien, dass der Abbau wohlfahrtsstaatlicher Leistungen seit der Finanzkrise 2008 vielerorts zu einem Anstieg von Ungleichheit zwischen und innerhalb von Städten geführt hat (Donald et al., 2014:7) und, dass die ,regressive redistribution“ kommunaler Dienstleistungen besonders die ohnehin sozial und ökonomisch benachteiligten Bevölkerungsgruppen betrifft (Hastings et al., 2017:2008)

\subsection{Digitale Neuordnung der Bibliothek als Raum}

Die Möglichkeiten der Digitalisierung haben die eingangs beschriebene Debatte darüber entfacht, wozu es Bibliotheken überhaupt noch braucht (Jochumsen et al., 2012:587). Michnik (2014:427) spricht von einem ,struggle for legitimacy“ für die Bibliothek als physischen Raum, der an vielen Standorten mit Kürzungs- und Schließungsdebatten einhergeht (Hobohm, 2013:623; Hvenegaard Rasmussen, 2016:549). Öffentliche Bibliotheken müssen sich an veränderte Lesegewohnheiten, den technologischen Fortschritt und wachsende Ansprüche der Nutzer*innen jenseits von Bücherausleihen anpassen. Dabei verändern sich die ihnen zugeschriebenen Funktionen als auch ihr Medienangebot und der Leistungszugang. In den letzten Jahren wurden daher vermehrt Anstrengungen unternommen, um das Angebot auszubauen und sichtbarer zu machen (Abb. 2), insbesondere durch den Einsatz von Informations- und Kommunikationstechnologien oder als digitale Bibliotheken mit gänzlich neuen Dienstleistungsangeboten. Der durch die Digitalisierung initiierte Wandel betrifft sowohl die Bibliothek als multifunktionalen Raum als auch ihre Rolle als Ort(e) in der Stadt.

\subsubsection{Die Bibliothek als multifunktionaler Raum}

Bibliotheken präsentieren und positionieren sich sowohl im physischen als auch im digital-virtuellen Raum als multimediale, hybride Orte des Lernens und der Innovation. Zusätzlich zu den klassischen Lesesälen findet man immer häufiger Lounge-Bereiche, die die Aufenthaltsqualität verbessern sollen. Gleichzeitig nutzen Bibliotheken E-LearningPortale (Stadtbibliothek Chemnitz, 2019) und Social-MediaPlattformen (Stadtbibliothek Bonn, 2019), um den Austausch mit ihrem Publikum zu vertiefen, setzen eigene Plattformen zur multimodalen Kommunikation auf (Stadtbibliothek Köln, 2019) und gehen neue Wege in der Wissensvermittlung (Vogt et al., 2016).

Im Zuge des informationstechnologischen Wandels findet auch eine Hinwendung zu kundenorientierten kommunikativen und partizipativen Strategien statt - „from collection to 


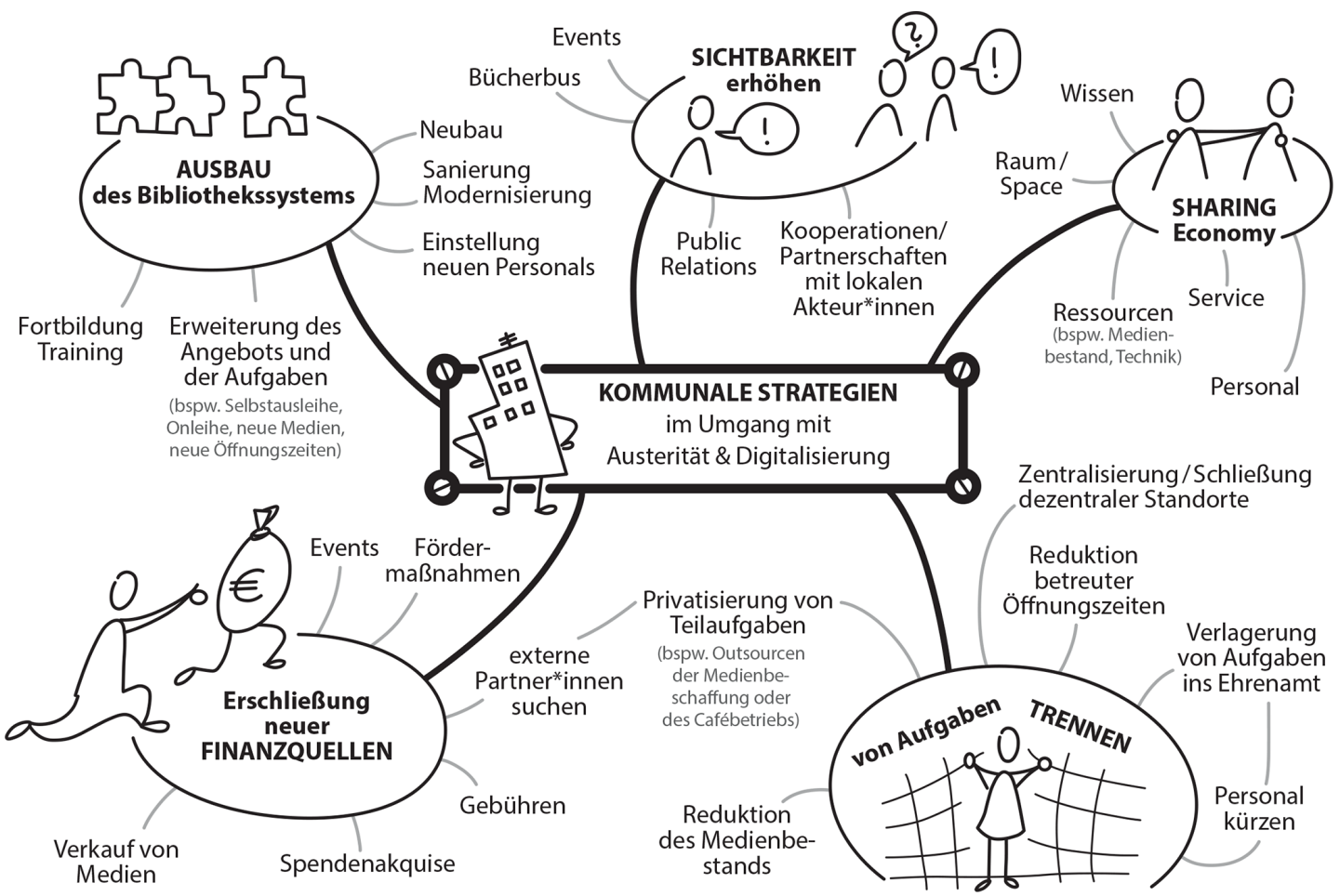

Abb. 2. Kommunale Strategien im Umgang mit Austerität und Digitalisierung. Grafik: Katja Thiele \& Irene Johannsen.

connection“ (Jochumsen et al., 2012:588) und „from access to user participation" (Hvenegaard Rasmussen, 2016:547). Dabei orientieren sich Bibliotheken an innovativen Konzepten wie FabLabs, LibraryLabs oder Makerspaces. Um diese Angebote zu ermöglichen, gehen sie, neben der klassischen Zusammenarbeit mit Bildungseinrichtungen wie Schulen und Universitäten, neue Kooperationsformen ein und öffnen sich Softwareentwickler*innen, Unternehmer*innen und der Do-it-yourself-Bewegung. In der Zentralbibliothek Köln können Nutzer*innen seit letztem Jahr Workshops besuchen, die ihnen ermöglichen, eigene Projekte weiterzuentwickeln, sich mit aktuellen Technologien (u.a. Coding, virtuelle Realität, 3-D-Modellierung -Druck) sowie Themen des digitalen Wandels (u.a. Schreiben für Blogger*innen) zu beschäftigen (Stadtbibliothek Köln, 2018).

Als Antwort auf veränderte Konsumbedürfnisse und, weil Bibliotheken gezwungen sind, stärker im Netz zu agieren und „Bibliotheksleistungen in abgestimmter Kooperation“ zu organisieren (Knoche, 2018:91), ist außerdem die Weiterentwicklung von Bibliotheken zu multifunktionalen Kulturund Veranstaltungsorten zu beobachten. Nutzer*innen können immer häufiger Virtual-Reality- und Gaming-Angebote wahrnehmen (Stadt Düsseldorf, 2019) und angesagte Events besuchen (z.B. Live-Escape-Game). Neben selbst organisierten Einzelveranstaltungen arbeiten kommunale Bibliotheken auch gemeinsam an der Realisierung längerer, größerer oder überlokaler Festivals und Themenwochen, teilweise in Ko- operation mit privaten Akteur*innen (Stadtbibliothek Köln, 2018; ATZE Musiktheater GmbH, 2019).

Während Bibliotheken auf der einen Seite stärker als dritte Orte - der Begegnung, der Interaktion - und partizipative Orte - des Mit-Machens, des Selber-Machens - verstanden und als Motor für Partizipation, Inklusion, Entwicklung, Innovation oder Wachstum visioniert werden (Di Marino und Lapintie, 2015; Hobohm, 2013, 2017), wird auf der anderen Seite bezweifelt, dass sich die damit verbundenen Versprechen auf Gerechtigkeit in der Realität einlösen lassen (Hvenegaard Rasmussen, 2016:551). Durch die Hinwendung von Bibliotheken zu kommunikativen und partizipativen Strategien werden zwar die Kooperation von Kommunen, die lokale Verankerung von Bibliotheken und deren Kooperation mit lokalen Akteur*innen gestärkt. Diese Entwicklung von Bibliotheken ist jedoch auch im Kontext zu den in Abschnitt 3.1 beschriebenen Logiken der Finanzknappheit zu sehen.

\subsubsection{Bibliotheken als Ort(e) in der Stadt}

Werfen wir den Blick nicht nur auf die Bibliothek selbst, sondern auf die Bedeutung der Bibliothek als Ort(e) in der Stadt, können wir beobachten, dass öffentliche Bibliotheken zur Hoffnungsträgerinnen wirtschaftlichen Aufschwungs und gesellschaftlicher Teilhabe geworden sind. Beispiele von spektakulären Bibliotheksgebäuden in Birmingham, Aarhus, Amsterdam oder Seattle (Jochumsen et al., 2012:587) zeigen, dass Stadtplaner*innen sie weltweit sogar ins Zentrum 
ihrer stadtentwicklungspolitischen Überlegungen stellen und von international renommierten Architekt*innen designen lassen. Ihre Bedeutung für die Aufwertung von Innenstädten zeigt sich schon an ihrer häufigen Nennung als Sehenswürdigkeiten auf Tourismus-Webseiten oder Lifestyle-Blogs (z.B. http://tripadvisor.de, letzter Zugriff: 15. Mai 2020). Die Aufmerksamkeit steigt auch in Deutschland, wo derartige Projekte bereits in Stuttgart (Sayah, 2011) oder Berlin (Bernau, 2020) umgesetzt wurden oder sich in der Planungsphase befinden. Auch die rege Zusammenarbeit von Städtebauförderung, Landesministerien und Bibliotheksverbänden im Rahmen regelmäßiger Konferenzen macht deutlich, ,dass Bibliotheken [als] kommunale Vorreiter im Zeichen digitaler kultureller Bildung" wahrgenommen werden (wollen) (BIB e.V., 2019). „Der aktuelle [...] Diskurs über [...] [das] Bibliothekssterben [...] [und das] Ende der Bibliothek [...] bei einer gleichzeitig zu beobachtenden Renaissance der gesellschaftlichen Investition in diese [...] [lässt] sogar vermuten, dass sie nie wirklich ausschließlich Informationseinrichtung waren“ (Hobohm, 2013:622). Öffentliche Bibliotheken werden gezielt eingesetzt, um im Städtewettbewerb Mittel einzuwerben (Carlsson, 2013), wobei besonders der architektonischen Hülle eine zentrale Bedeutung zugeschrieben wird. Während Leiter*innen und Angestellte öffentlicher Bibliotheken sich mehr Aufmerksamkeit (und finanzielle Mittel) für ihre Einrichtungen wünschen, verbinden politische Akteur*innen mit ihnen auch die Möglichkeit, „Leuchttürme“ in der eigenen Stadt zu platzieren, die wiederum Aufmerksamkeit im (inter)nationalen Städtewettbewerb erzeugen. ,[A]s an answer to strategies of culture-led urban regeneration" (Carlsson, 2013:7) sollen sie als Ikonen der Stadtentwicklung dienen (Skot-Hansen et al., 2013:10), das ,place-making“ befördern (Skot-Hansen et al., 2013:12f.) und lokale Identität, Innovation und Kohäsion stärken (Skot-Hansen et al., 2013:13ff.).

In Bezug auf die digitale Neuordnung der Bibliothek als Raum lässt sich festhalten, dass (öffentliche) Bibliotheken aufgrund ihrer Nähe zur Medienentwicklung im Vergleich zu anderen sozialen Infrastrukturen stärker von der Digitalisierung profitieren (u.a. mehr Sichtbarkeit, Aufwertung einzelner Standorte). Als freiwillige kommunale Leistungen stehen sie jedoch auch im Fokus kommunaler Austerität. Dass dabei auf den ersten Blick widersprüchliche Strategien im Umgang mit Austerität und Digitalisierung ineinandergreifen, zeigt das Beispiel der jahrelangen Diskussion um die Zentral- und Landesbibliothek (ZLB) in Berlin. Die Modernisierung der ZLB wird von verschiedenen Akteur*innen bereits seit den 1980er Jahren gefordert und in Teilen umgesetzt. 2019 ist die ZLB deshalb vom Deutschen Bibliotheksverband zur Bibliothek des Jahres gewählt worden, ausgezeichnet für die Umsetzung innovativer Angebote (u.a. Ausleihe von Ölgemälden oder Noten) und Veranstaltungskonzepte (u.a. Schattentheater, Makerspace) (Kufner, 2019). Das weiterhin zur Diskussion stehende Konzept eines ,,baulich gewaltigen Kulturzentrums“ (Bernau, 2020) ist jedoch stark umstritten, wegen der möglichen Folgen eines monumentalen Neubaus für das städtebauliche Umfeld (Breher, 2020) sowie der damit verbundenen Kosten (Stollowsky und Kurpjuweit, 2014). Steigende Ausgaben für technische Innovationen machen (bei gleichzeitig sinkendem kommunalen Budget) außerdem Kürzungen an anderer Stelle nötig (bspw. Outsourcen der Literaturauswahl an einen externen Bibliotheksdienstleister aus Reutlingen; Rada, 2014). So wird die Einführung moderner Technologien mit lokalen austeritätspolitischen Strategien verbunden (Pollio, 2016). Dass öffentliche Bibliotheken zunehmend unter Druck geraten, neue (multimediale) Angebote zu schaffen, ist besonders für kleine bzw. dezentrale Bibliotheken mit geringen Ressourcen problematisch. Als Reaktion darauf entstanden bereits in den letzten 20 Jahren vielerorts verschiedene Formen nicht-staatlicher Angebote (bspw. Bücherbusse, Büchertauschbörsen, Ehrenamtsmodelle etc.) (Seefeld, 2018). Diese lassen sich sowohl als Minimallösungen im Umgang mit staatlicher Unterfinanzierung als auch als zivilgesellschaftliche Antwort auf diese deuten, wobei ,,bürgerschaftliches Engagement [...] vom Staat [immer wieder] zur Aufrechterhaltung öffentlicher Leistungen instrumentalisiert" wird (van Dyk und Haubner, 2019:266), um Protesten gegen Schließungen vorzubeugen oder einen weiteren Rückzug des Sozialstaats zu legitimieren. Die Ambivalenz dieser Entwicklung wird nachfolgend anhand eigener empirischer Ergebnisse in der Stadt Bonn diskutiert.

\section{Ambivalenzen kommunaler Bibliothekspolitik in Bonn}

\subsection{Methodik und Daten}

Aufbauend auf den Erkenntnissen der Austeritäts- und Infrastrukturforschung (u.a. Brenner und Theodore, 2002; Peck, 2015; Schipper und Schönig, 2016; Wissen und Naumann, 2008) ist davon auszugehen, dass Governance-Strukturen, Akteur*innenkonstellationen und politische Regimebildungen im Bereich sozialer Infrastrukturen lediglich in ihren spezifischen geographischen Settings verstanden werden können, da die Veränderung politischer Rationalitäten und der Umbau sozialer Daseinsvorsorge auf der lokalen Ebene besonders sichtbar werden (Featherstone et al., 2012). Die Transformation öffentlicher Bibliotheken wird deshalb ausgehend von der lokalen Ebene in den Blick genommen und in Bezug auf die Auswirkungen von Austeritäts- und Digitalisierungspolitiken auf die Angebotsstrukturen untersucht. Im Fokus steht die Frage, welche Veränderungen und sozial-räumlichen Implikationen dadurch ausgelöst werden. Vor dem Hintergrund der Debatten um Austerity Urbanism (Peck, 2012, 2015) wird so ein Beitrag zur Beantwortung der Frage geleistet, wie politische Restrukturierungsprozesse die gesellschaftlichen Verhältnisse (hier: die kommunale Daseinsvorsorge am Beispiel öffentlicher Bibliotheken) verändern. Das hilft, die lokalen Ausprägungen von Austerität besser zu verstehen und die Debatten um die Entstehung lokaler Regimekonstellationen unter Austeritätsbedingungen 
(Davies und Blanco, 2017; Featherstone et al., 2012) zu bereichern.

Veränderungen in der Bibliothekslandschaft lassen sich in Städten verschiedener Größe beobachten. Die Veränderungen der Angebotsstruktur im kommunalen Bibliotheksnetz lassen sich jedoch besonders deutlich am Beispiel von Großstädten darstellen, da diese mehr als einen Bibliotheksstandort unterhalten und die Neuorganisation das gesamte Bibliothekswesen (inklusive der Personalstruktur) einer Kommune betrifft. Um die Relevanz jener Entwicklungen für die deutsche Debatte um Daseinsvorsorge (Mattert et al., 2017) aufzuzeigen, konzentriert sich dieser Artikel auf die Ergebnisse einer Fallstudie in der Stadt Bonn, die zwischen 20162019 im Rahmen eines europäischen Vergleichs ${ }^{4}$ von Fallstudien in Bonn (D), Leicester (UK) und Malmö (S) erhoben wurden. In den Abschnitten 4.3 bis 4.5 werden zentrale Strategien der Kommunen zur Weiterentwicklung ihres Bibliothekswesens am Beispiel der Stadt Bonn dargestellt und hinsichtlich ihrer Wirkungen beleuchtet. Auf die städtischen Kontextbedingungen und die Übertragbarkeit der Ergebnisse innerhalb des deutschen Kontextes wird in Abschnitt 4.2 Bezug genommen.

Im Sinne der Triangulation von Verfahren (Flick, 2004:12) wurde für die Datenerhebung in Bonn ein qualitativer Methoden-Mix angestrebt. Dieser umfasst die inhaltsanalytische Auswertung von relevanten Dokumenten und Daten, teilnehmenden Beobachtungen und qualitativen LeitfadenInterviews mit Expert*innen ${ }^{5}$.

Interviewt wurden im Zeitraum von 2017-2019 15 relevante Akteur*innen im Feld der kommunalen Bibliothekspolitik, d.h. Funktionsträger*innen aus den beforschten Einrichtungen und ihren Trägerorganisationen, Vertreter*innen der kommunalen Politik und des Landes Nordrhein-Westfalen (NRW), der Verwaltung und von Verbänden, Organisationen und Organen des lokalen und nationalen Bibliothekswesens (Deutscher Bibliotheksverband e.V.; Berufsverband Information Bibliothek e.V.; Fachstel-

\footnotetext{
${ }^{4}$ Die hier publizierten Ergebnisse sind Teil des durch die Deutsche Forschungsgemeinschaft (DFG) geförderten Forschungsvorhabens „Öffentliche Bibliotheken im Spannungsfeld von Finanzknappheit und kommunaler Daseinsvorsorge“. Ausgehend von einer Mehrebenen-Perspektive berücksichtigt das Projekt auch die Rolle nationaler und weiterer nichtlokaler Akteur*innen und Institutionen für die lokalen Entwicklungen. Ein Vergleich mehrerer lokaler Fallstudien in unterschiedlichen nationalen Kontexten ermöglicht es, die Bedeutung von nationalen Unterschieden im Wohlfahrtsstaat (welfare states, Esping-Andersen, 1990; geographies of actually existing neoliberalism, Brenner und Theodore, 2002:349) zu beleuchten. Darauf kann hier jedoch nicht näher eingegangen werden.

${ }^{5}$ Das verwendete Interviewmaterial wird nachfolgend im Text durch ,(Interviews)“ ausgewiesen. Die Interviews werden nicht direkt zugeordnet, da sich alle Aussagen im Text auf Aussagen aus mehr als einem Interview beziehen. Dies dient zudem der Anonymisierung.
}

le für Öffentliche Bibliotheken NRW) sowie der Zivilgesellschaft (Fördervereine der Bonner Bibliotheksstandorte und Bürger*inneninitiativen). In die ergänzende Analyse von Dokumenten einbezogen wurden Publikationen jener Akteur*innen (Berichte der kommunalen Körperschaften, Veröffentlichungen von Verbänden, Organisationen und Organen des Bibliothekswesens, öffentliche Stellungnahmen politischer Parteien und Veröffentlichungen zivilgesellschaftlicher Initiativen) und Zeitungsartikel der täglich erscheinenden Lokalzeitung (General Anzeiger Bonn), die über aktuelle Entwicklungen im Bibliothekswesen im Zeitraum von 1. Januar 2008-31. Januar 2017 berichteten. Die Interviewtranskripte und Materialien wurden nach dem Prinzip der qualitativen Inhaltsanalyse nach Mayring und Fenzl (2019:640) hinsichtlich folgender zuvor festgelegter und aus der Theorie abgeleiteter Analysekriterien untersucht: (1) Rechtliche Regelungen und Finanzierungsstrukturen auf nichtlokalen Maßstabsebenen, (2) Funktionen von Bibliotheken, (3) organisatorische Veränderungen und kommunale Strategien und (4) ungleiche Entwicklung von Bibliothekslandschaften.

Um das in den Interviews gewonnene Fakten- und Kontextwissen der Akteur*innen durch überindividuelles Kontextwissen (,contextual understanding“, Kearns, 2000:105f.) zu ergänzen, wurden zusätzlich teilnehmende Beobachtungen bei ausgewählten Veranstaltungen in die Datenerhebung integriert. Dazu gehörte bspw. die aktive Teilnahme am jährlichen bundesweiten Deutschen Bibliothekskongress sowie an Vernetzungstreffen relevanter Akteur*innen. Ebenfalls in die Ergebnisse eingeflossen sind einfache Auswertungen statistischer Daten zuständiger Behörden (Deutsche Bibliotheksstatistik, Statistikstelle Bonn) zu den städtischen (Kontext-)Bedingungen, den Veränderungen des lokalen Bibliothekssystems sowie den institutionellen Bedingungen (bspw. Mittelverteilung) auf nationaler Ebene.

\subsection{Fallstudienkontext}

Bonn ist eine kreisfreie Großstadt mit 331079 Einwohner*innen, liegt im Regierungsbezirk Köln und gehört zu den wachsenden Großstädten in NRW (Bundesstadt Bonn, 2019). Als traditionsreiche Universitätsstadt hat sie einen hohen Anteil von Akademiker*innen (28,5\%, 30. Juni 2017) und, im Vergleich zu anderen Städten, eine relativ geringe Arbeitslosenquote (Bundesstadt Bonn, 2019). Das öffentlich-kommunale Bibliothekssystem besteht neben einer Vielzahl kirchlicher Büchereien - aus neun öffentlichen Stadtteilbibliotheken. In Bezug auf die Finanzierung der kommunalen Bibliotheken stellt Bonn aufgrund seiner historischen Bedeutung als Bundeshauptstadt zwischen 1949-1990 gewissermaßen einen Sonderfall dar, denn als ehemalige Bundeshauptstadt konnte die Stadt bis ins Jahr 2010 auf besondere finanzielle Förderungen durch den Bund zurückgreifen (Bundesstadt Bonn, 2013:4). Seit 2010 muss Bonn als Bundesstadt „die Aufwendungen für die 
städtische Kultur im Wesentlichen wieder allein [tragen]“ (Bundesstadt Bonn, 2013). Hinzu kommt, dass sich die Stadt aufgrund hoher Schulden in Folge des Skandals um den Bau des World Conference Centers Bonn (WCCB) seit einigen Jahren im Haushaltssicherungskonzept des Landes NRW befindet, d.h. die Kommunalaufsicht in Köln den Haushalt in Bonn genehmigen muss (Akalin et al., 2012). Dies hat erhebliche Auswirkungen auf die Ausgestaltung der Bonner Kultur und damit auf das öffentliche Bibliothekswesen. In Bonn wird daher schon seit der Jahrtausendwende die Frage diskutiert, in welchem Maße sich die Stadt kommunale Dienstleistungen leisten will und kann. Davon waren besonders die öffentlichen Stadtteilbibliotheken betroffen, wobei sich gegen Mittelkürzungen und geplante Schließungen zivilgesellschaftlicher Widerstand formierte und Fördervereine gegründet wurden (Abb. 3). Unabhängig davon wurde ab 2006 das Haus der Bildung als neuer zentraler Standort der Stadtbibliothek geplant und 2015 eröffnet. Der (umstrittene) Umgang mit den Stadtteilbibliotheken wird im seit 2012 entwickelten und seit 2016 umgesetzten neuen Bibliothekskonzept geregelt.

Im Vergleich zu deutschen Städten gleicher Größe nimmt Bonn aufgrund seiner historischen Bedeutung zwar eine Sonderrolle in Bezug auf die finanzielle Ausstattung ein. Es lassen sich dennoch Veränderungen und kommunale Strategien erkennen, die auch in zahlreichen anderen deutschen Städten (u.a. Düsseldorf, Duisburg, Berlin) beobachtet werden können (bspw. Schließungen von dezentralen Bibliotheksstandorten, eklatante Mittelkürzungen; DBS). Nachfolgend wird verdeutlicht, wie in Bonn die Herausforderungen von Austerität und Digitalisierung eine Strategie beförderten, die zur Verschärfung sozial-räumlicher Disparitäten zwischen zentralen und dezentralen Standorten geführt hat.

\subsection{Zentralisierung von Bibliotheksangeboten im neuen Haus der Bildung}

Anfang des Jahrtausends stand die Stadt Bonn, wie zahlreiche Kommunen in Deutschland, vor dem Problem, dass durch kontinuierlich sinkende kommunale Investitionstätigkeit im Bereich Infrastrukturen (Reidenbach et al., 2008) die zentrale Stadtbibliothek in einem desolaten Zustand war: die baulichen Strukturen waren marode und es herrschte Platzmangel. Die Situation der Zentralbibliothek wurde von allen Beteiligten im parlamentarischen Prozess deshalb als schlecht bewertet. Nach einer langen stadtpolitischen Kontroverse, die hier nicht detailliert wiedergegeben werden kann, entschied sich der Rat 2006 gegen einen vollständigen Neubau und für eine Kombination aus Sanierung und Neubau in zentraler Innenstadtlage am bisherigen Standort (Interviews) (Abb. 3). Im neuen Haus der Bildung sollten Volkshochschule (VHS) und Zentralbibliothek an einem Standort integriert werden. Als Argument dafür wurde vor allem die, im Kontext der Digitalisierung als notwendig angesehene, Modernisierung und der Umbau der
Bibliothek im Sinne eines „dritten Ortes“ angeführt (Interviews). Die Bonner Bibliotheken dürften die zukünftige Entwicklung nicht verpassen und müssten zwar den Bestand erhalten, aber auch digitale Angebote schaffen und „Besonderes“ anbieten (Interviews). Ein gemeinsames Gebäude mit der VHS versprach zusätzlich Synergie-Effekte: Durch räumlich-funktionale und inhaltlich-thematische Kooperationen sollten erstens die verschiedenen Zielgruppen stärker vernetzt werden (Bundesstadt Bonn, 2010). Zweitens sollte die Ansiedlung inmitten der Innenstadt und in unmittelbarer Bahnhofsnähe die Sichtbarkeit für beide Einrichtungen erhöhen und als Standortfaktor für die gesamte Stadt fungieren (Bundesstadt Bonn, 2010). Damit einher ging die Hoffnung, durch eine bessere Erreichbarkeit neue Besucher*innen aus dem Umland anzuziehen sowie die Umsätze der umliegenden Geschäfte zu erhöhen und damit Anreize für potentielle Investor*innen zu schaffen (Interviews).

Knapp 10 Jahre nach Planungsbeginn wurde das Haus der Bildung im Jahr 2015 fertiggestellt. Investiert wurden 26,4 Millionen Euro aus Mitteln der Kommune und des Landes NRW. Entstanden ist ein einladendes Eingangsgebäude mit repräsentativem Lese- und Lichthof, zahlreichen technischen Neuerungen (u.a. Mediennischen, ein Selbstverbuchungssystem, Ausleihautomaten und neue WLAN-Angebote) sowie attraktiven Aufenthalts- und Kommunikationsräumen (Inhoffen, 2015; Interviews). Diese Veränderungen im Zuge der Digitalisierung haben sowohl für die Stadtbibliothek als auch die VHS zu einer erhöhten Wahrnehmung ihrer Dienste geführt. Allein von 2016 auf 2017 ist die Anzahl der Medienausleihen (analog sowie E-Medien) in den Bonner kommunalen Bibliotheken und vor allem in der Zentralbibliothek stark gestiegen (Bundesstadt Bonn, 2017:3, 2018:5). Besonders die Zentralbibliothek hat eine deutliche Aufwertung erfahren und sich als Treffpunkt und gut besuchter Bildungs- und Informationsveranstaltungsort etabliert (Bundesstadt Bonn, 2017:7f.). In diesem Zusammenhang haben sich auch die Öffnungszeiten der Zentralbibliothek verlängert. Der zusätzliche Ausbau des Systems der „Onleihe“, d.h. die Möglichkeit Medien (Literatur, Hörbücher, DVDs etc.) über einen digitalen Katalog vorzumerken oder auszuleihen, macht das Angebot rund um die Uhr von zuhause aus digital verfügbar. Öffentliche Bibliotheken orientieren sich hierbei am Ideal der digitalen Gesellschaft und schaffen innovative Online-Angebote (bspw. eigene Streaming-Plattformen).

\subsection{Ausdünnung des Bonner Bibliotheksnetzes: das neue Bibliothekskonzept}

Ein Blick auf das Bibliothekssystem als Ganzes, also auf das Netz der öffentlichen Bibliotheken, zeigt, dass die Diskussionen und Entwicklungen um das Haus der Bildung in direktem Zusammenhang mit Einsparungen an sozialen Infrastrukturen in der Fläche stehen. Bereits seit Anfang des Jahrtausends wurde die Bibliothekspolitik in Bonn von einer Kontroverse über die Finanzierung der bestehenden Struk- 


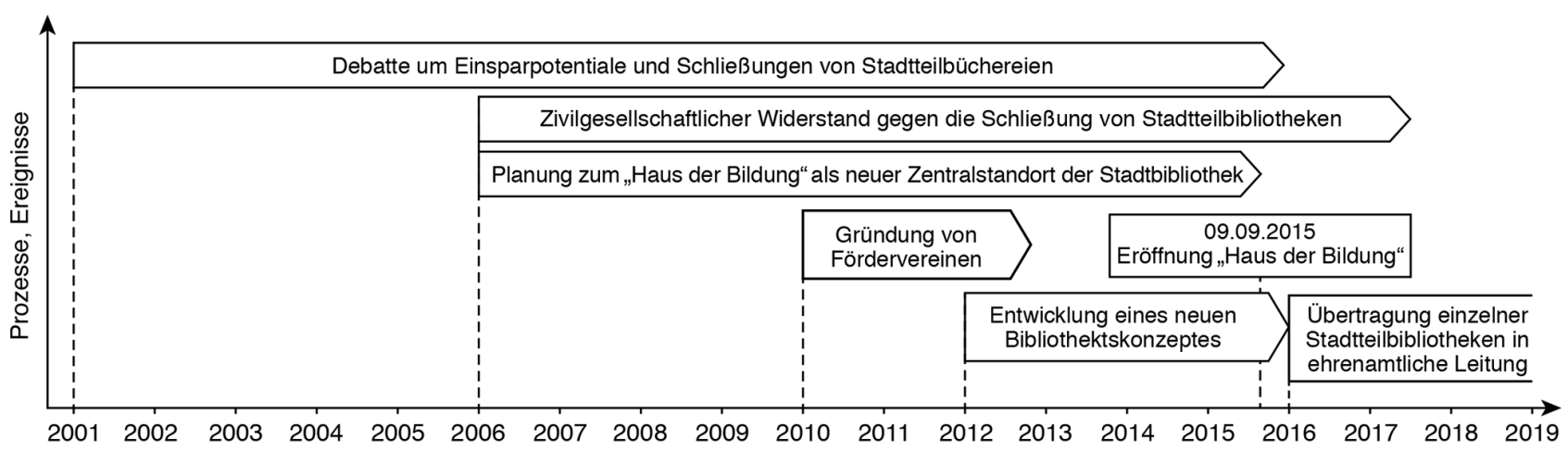

Abb. 3. Zeitstrahl Bonn. Grafik: Katja Thiele \& Irene Johannsen.

turen begleitet. Im Hintergrund standen damals wie heute die hohe Verschuldung der Stadt, Unstimmigkeiten zwischen den politischen Vertreter*innen bezüglich der Standorte und die Frage nach potentiellen Zuschüssen durch das Land NRW (Interviews). Um die Kosten zu senken, wurde immer wieder über Möglichkeiten der Einsparung von Personal, die Einführung einer Gebührenordnung, die Notwendigkeit neuer Kooperationen mit privaten Akteur*innen, die Einwerbung externer Mittel, die Verlagerung oder Auflösung von (Teil-)Beständen sowie die Schließung von Bezirks- und Stadtteilbibliotheken diskutiert (Inhoffen, 2014; Interviews).

Im Auftrag des Stadtrates entstand in den letzten drei Jahren vor der Fertigstellung des Hauses der Bildung (20122015) zudem ein neues Kulturkonzept zur Ausgestaltung der Kultur in der Fläche (Bundesstadt Bonn, 2013). In diesem Zusammenhang wurde auch ein Konzept für das Bonner Bibliotheksnetz entwickelt (Bundesstadt Bonn, 2014a). Es „,beschreibt zum einen die fachlich wünschenswerte Zielvorstellung [...], stellt dem aber gegenüber, wie unter Würdigung der seinerzeitigen finanziellen Situation der Stadt Bonn eine ressourcenschonende, strukturerhaltende Weiterentwicklung der Stadtbibliothek aussehen soll [...]“ (Bundesstadt Bonn, 2014b). Abschließend sah das Konzept daher eine Zentralisierung der räumlichen Struktur vor, d.h. die Stärkung einer zentralen Einrichtung bei gleichzeitigem Abbau dezentraler Einrichtungen (Bundesstadt Bonn, 2014b). Statt dem Erhalt der existierenden acht dezentralen Einrichtungen wurde nur noch eine Bibliothek pro Bezirk eingeplant, was das vorläufige Ende für die Standorte Bonn-Rheindorf (späterer Umzug in die heutige Integrierte Stadtteilbibliothek BonnAuerberg), Bonn-Dottendorf, Bonn-Endenich und Beuel-Ost bedeutete (Abb. 4). Damit sollte ein jährliches Einsparpotenzial in Höhe von EUR 444791 realisiert und durch die Einführung von Selbstverbuchungs- und Kassenautomaten zudem die Wirtschaftlichkeit des Bibliotheksbetriebes verbessert und die Personalkosten gesenkt werden (Bundesstadt Bonn, 2014b).

Nun könnte man davon ausgehen, dass moderne Bibliotheksysteme im digitalen Zeitalter in der Lage sind, einen
Verlust von Standorten in der Fläche durch die Erweiterung des digitalen Angebots auszugleichen und bspw. das System der „Onleihe“ durchaus zu einer besseren Versorgung in der Fläche beiträgt. Dies stimmt insofern, dass dadurch Nutzer*innen profitieren, die aufgrund einer zu großen Entfernung oder eingeschränkter Mobilität bisher keinen $\mathrm{Zu}-$ gang hatten. Die eigene Empirie hat jedoch auch gezeigt, dass der physische Zugang dadurch an einzelnen Standorten gefährdet ist (Interviews). Im Zuge der Zentralisierung übernimmt die Zentralbibliothek Sonderfunktionen beim Ausbau digitaler Angebote, wodurch jedoch der Druck auf dezentrale Bibliotheken in den Stadtteilen erhöht wird: Stadtteilbibliotheken übernehmen weiterhin vor allem soziale Funktionen und dienen den Menschen vor Ort über die reine Ausleihe hinaus als öffentlicher Raum, Treffpunkt, Begegnungsund Inspirationsort (Abb. 1). Diese Funktion steht jedoch in Konkurrenz zur Einführung technischer Lösungen (und deren Kosten); denn, wenn im Zuge von Haushaltseinsparungen dezentrale Stadtteilbibliotheken geschlossen oder Angebote ausgelagert werden, dann fallen wichtige physische Orte als Anker der sozialen Integration weg und die Daseinsvorsorgefunktion von Bibliotheken wird räumlich eingeschränkt (Interviews).

\subsection{Neuorganisation der (dezentralen) Stadtteilbibliotheken: Gefährdung öffentlicher Daseinsvorsorge durch Ehrenamt}

Das Bibliothekskonzept der Stadt Bonn war zum Zeitpunkt seiner Entstehung sowohl zwischen den politischen Parteien und innerhalb der Fraktionen als auch unter Mitgliedern der Verwaltung und zivilgesellschaftlichen Akteur*innen umstritten (Bundesstadt Bonn, 2014c; Heinz, 2015). Insbesondere Initiativen von Bürger*innen, die sich für den Erhalt einzelner Stadtteilbibliotheken einsetzten, sahen die "Grundversorgung in den Ortszentren" bedroht (Bundesstadt Bonn, 2014d). Aufgrund des zivilgesellschaftlichen Widerstands kam es nicht zur vollständigen Schließung der vier zur Schließung vorgesehenen Stadtteilbibliotheken. Viel- 


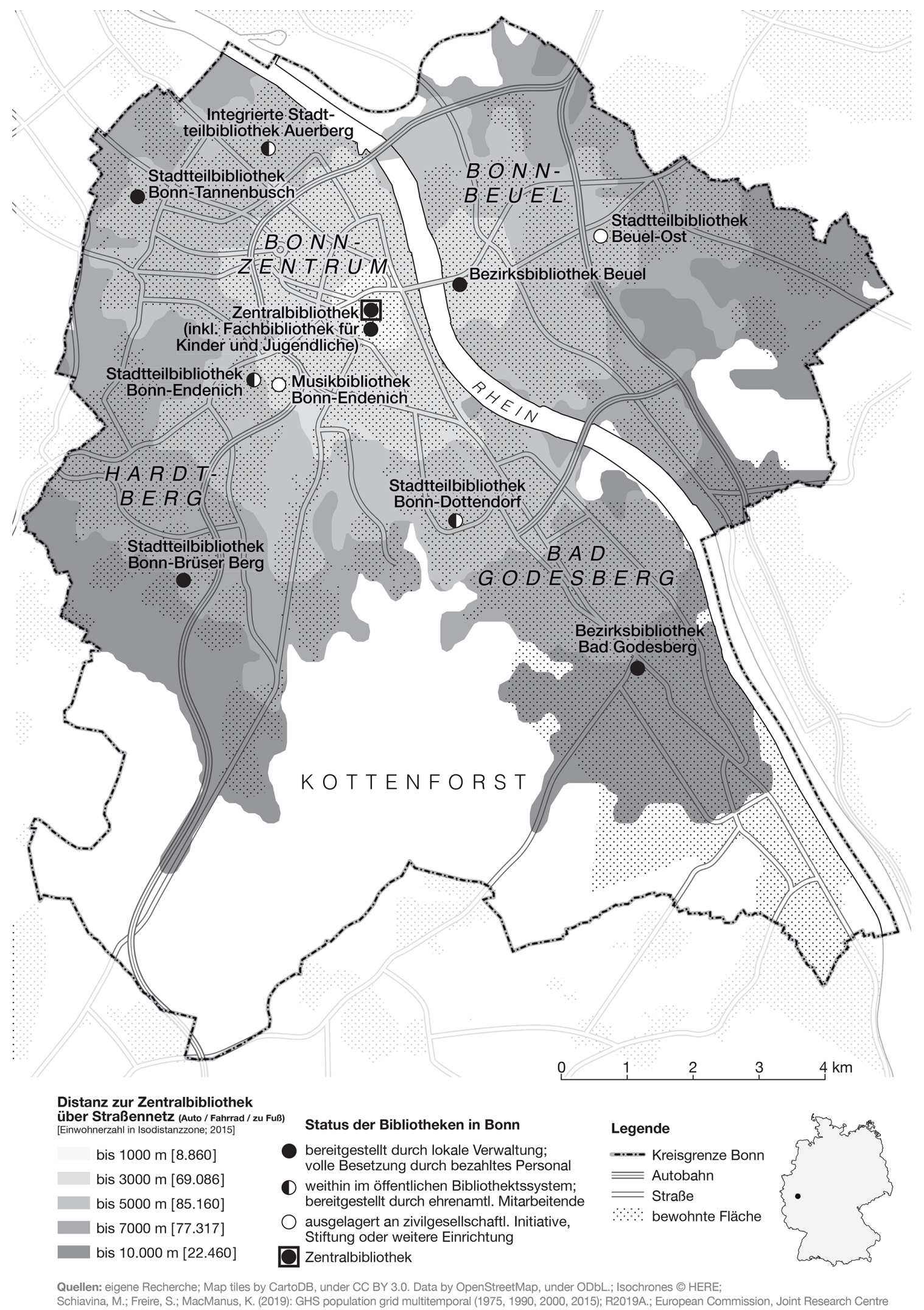

Abb. 4. Bibliotheken in Bonn und Entfernung zur Zentralbibliothek. Grafik: Irene Johannsen. 
mehr wurden in Abhängigkeit von den lokalen Kontexten (Stärke des lokalen Engagements, Bedarf, historisch gewachsene Strukturen) unterschiedliche Lösungen für die Standorte gefunden. In Bonn-Beuel wurde nach einer KostenNutzen-Abwägung der kleinere Standort Beuel-Ost in eine Schulbibliothek überführt, der größere und zentralere Standort verblieb in hauptamtlicher Leitung (Willcke, 2015). In den Stadtteilen Endenich und Dottendorf wurden hingegen Kooperations-Modelle umgesetzt, bei denen die materielle Infrastruktur (Gebäude, sanitäre Einrichtungen, ITInfrastruktur etc.) weiterhin durch die Stadt Bonn getragen wird, das Personal und die Organisation des Ausleihbetriebes vor Ort aber in die Hände ehrenamtlicher Fördervereine übertragen wurde (Knopp, 2015). Im Fall der Stadtteilbibliothek in Bonn-Rheindorf wurde ein Vertrag mit einer sozialen Organisation aus dem nahe gelegenen Stadtteil Auerberg geschlossen, die als Trägerverein die Leitung einer integrativen Bibliothek - es werden neben Ehrenamtlichen auch Menschen mit Behinderungen in die Bibliotheksarbeit integriert übernimmt (Knopp, 2015). Hier liegen sowohl die Erhaltung der materiellen Strukturen als auch die Finanzierung und Organisation des Personals in der Verantwortung des Vereins.

Interessant ist, dass trotz finanzieller Notlage am Ende des politischen Prozesses nicht die Schließung von Stadtteilbibliotheken stand. Es könnte sogar von einer Stärkung der lokalen Strukturen durch die intensive Mobilisierung der betroffenen Bevölkerung für ihre Bibliothek und damit für die Lebendigkeit der Stadtteile gesprochen werden. In Bezug auf die räumliche Struktur des Bibliotheksnetzes muss jedoch differenziert werden. Während das Angebot in der Zentralbibliothek ausgebaut und durch die Modernisierung auf den neuesten Stand (der Technik) gebracht wird, können in den Stadtteilbibliotheken zwar weiterhin Veranstaltungen besucht und Bibliotheksservices genutzt werden; spezielle und innovative Angebote sind jedoch selten(er) verfügbar. Im Alltag bedeutet das für die betroffenen Nutzer*innen eine Mehrbelastung (bspw. durch weitere Anfahrtswege zur Zentralbibliothek, Abb. 4). Hiervon sind mobilitätseingeschränkte und einkommensschwache Gruppen am stärksten betroffen. Hinzu kommt, dass auch nach den Umstrukturierungen permanent über Kürzungen im Kleinen diskutiert wird (u.a. Raumkapazitäten, Anzahl und Ausstattung der Computerarbeitsplätze, zur Verfügung stehender Bestand an Medien, Verringerung von durch Personal betreute Öffnungszeiten), die die Bibliotheksarbeit in den Stadtteilen erschweren (Interviews).

Der unterschiedliche Ausgang der Debatten um die einzelnen Stadtteilbibliotheken in Bonn verweist zudem auf ein unterschiedlich starkes Engagement in den Stadtteilen, was auch zurückzuführen ist auf ungleiche Möglichkeiten der betroffenen Nutzer*innen, politische Entscheidungsprozesse zu beeinflussen. Besonders problematisch ist, dass ehrenamtliche Arbeit und damit die Quantität und Qualität der Angebote stark von einzelnen Personen abhängen und großen Schwankungen ausgesetzt ist (Interviews).
Da Ehrenamtler*innen (oder automatisierte Ausleihsysteme) kein professionell ausgebildetes Personal ersetzen können, ist eine Umstellung auf ehrenamtliche Strukturen auch in der Fachwelt umstritten (dbv e.V., 2014). Daher muss festgestellt werden, dass sich die Förderung bzw. Nutzung freiwilligen Engagements für öffentliche Bibliotheken (und andere Bereiche der sozialen Daseinsvorsorge) nicht als einfache Lösung, sondern als ambivalent darstellt (Pinl, 2015:52f.). Durch eine Zentralisierung der räumlichen Struktur werden kommunale Bibliothekssysteme langfristig ausgedünnt, eingeschränkt und ihre nachhaltige Gewährleistung gefährdet. Ehrenamtliches Engagement wird genutzt, um Kürzungseffekte temporär abzumildern; einmal ausgelagerte Verantwortung und der Verlust beruflicher Kompetenzen lassen sich in der Praxis jedoch nur schwer wieder rückgängig machen (Pinl, 2015:52f.) und die Grenzen zwischen Partizipation, Widerstand und Selbsthilfe verschwimmen.

\section{Fazit und Schlussfolgerungen}

Vor dem Hintergrund der Debatten um den Einfluss von Austerität und Digitalisierung auf lokale soziale Infrastrukturen hat der Beitrag gezeigt, dass öffentliche Bibliotheken ein spannender Gegenstand für die geographische Stadtforschung sind.

Im Zuge von Digitalisierung und Austerität(spolitik) finden im öffentlichen Bibliothekswesen große Veränderungen statt: Die Digitalisierung stellt Bibliotheken vor neue Herausforderungen im Hinblick auf die Erreichung alter und neuer Zielgruppen, die Veränderung von Funktionen und Angeboten und ihre Neuerfindung als Raum - sowohl intern als auch als Ort(e) in der Stadt. Gleichzeitig stehen Kommunen vor dem Problem der Finanzknappheit, von der freiwillige Leistungen der Kommunen besonders betroffen sind, und müssen ihre Bibliothekspolitik daran anpassen. Die sich verändernden Funktionen von Bibliotheken sind abhängig von den gesellschaftlichen Vorstellungen der beteiligten Akteur*innen, was Bibliotheken leisten können (und sollen) und sind verbunden mit den sich ebenfalls wandelnden Verständnissen von Bibliotheken als Räume. Der Beitrag hat die zentralen Strategien dargestellt, mit denen Kommunen auf die Veränderungen von Bibliotheken reagieren und die räumlichen Strukturen, die interne Organisation, die Personalausstattung und deren Finanzierung anpassen. Er hat auch gezeigt, dass Maßnahmen im Kontext von Digitalisierungsstrategien sich oft nicht klar trennen lassen von austeritätspolitischen Maßnahmen, da diese häufig ineinandergreifen. Mit jenen Veränderungen ist wiederum die ungleiche Entwicklung von Bibliotheks- und Bildungslandschaften, konkret ihrer sozialen und räumlichen Strukturen, verbunden. Die eingangs angeführte These, dass die Digitalisierung für Bibliotheken eine Verbesserung des Angebots in der Breite befördert, kann nur teilweise bestätigt werden. 
In Bonn lässt sich eine Tendenz zur Zentralisierung von räumlichen Strukturen bei einer gleichzeitigen Ausdünnung dezentraler Standorte und der Verlagerung städtischer Aufgaben in ehrenamtliche Verantwortung erkennen. Dadurch wird die Repräsentationsfunktion öffentlicher Bibliotheken an zentralen Standorten gestärkt. Dies hat jedoch negative Auswirkungen auf Angebot und Zugang in der Fläche. Unter den Bedingungen von Austeritätspolitik führen Digitalisierungsstrategien nicht dazu, die besonders wichtigen analogen Angebote öffentlicher Bibliotheken in der Fläche zu erhalten oder auszuweiten. Durch die Fokussierung auf einen zentralen „Leuchtturm“ der Bibliothekspolitik im Zentrum der Stadt geraten soziale Funktionen in anderen Teilen der Stadt verstärkt unter Druck (bspw. die der Teilhabe, des Aufenthaltes, der Begegnung, der Breite des Angebotes etc.) und es kommt zu einer räumlichen Verlagerung von Angeboten der sozialen Infrastruktur und zu einer Verschlechterung der Bildungsgerechtigkeit im Sinne einer breiten, dezentralen Versorgung. Diese Entwicklung der Angebotsstruktur schränkt den Zugang vor allem für mobilitätseingeschränkte und einkommensschwache Bevölkerungsgruppen ein. Auch die Erweiterung des digitalen Angebots (bspw. „Onleihe“) kann die sozialen Funktionen von Stadtteilbibliotheken an dezentralen Standorten nur teilweise auffangen, denn für die tägliche Daseinsvorsorge und als Anker der sozialen Integration im Stadtteil übernehmen gerade die physischen, dezentralen Standorte eine entscheidende Rolle. Inwiefern noch von einer zeitlichen und räumlichen Gleichwertigkeit dieser Angebote ausgegangen werden kann, wenn Angebote der öffentlichen Daseinsvorsorge an Fremddienstleister*innen ausgelagert oder durch ehrenamtliche Strukturen getragen werden, ist fraglich und bedarf weiterer kritisch-geographischer Reflektionen.

Datenverfügbarkeit. Dieser Artikel basiert auf empirischer Forschung der Autorin im Rahmen ihrer Promotion. Die Daten sind daher bisher nicht öffentlich zugänglich.

Interessenkonflikt. Die Autorin erklärt, dass kein Interessenkonflikt besteht.

Danksagung. Ich bedanke mich bei Britta Klagge und Nele Steffen sowie bei den anonymen Gutachter*innen für das konstruktive Feedback. Die Forschung zu diesem Artikel wurde von der Deutschen Forschungsgemeinschaft finanziert.

Finanzierung. This research has been supported by the Deutsche Forschungsgemeinschaft (grant no. 424608044).

Begutachtung. This paper was edited by Benedikt Korf and reviewed by three anonymous referees.

\section{Literatur}

Aabø, S. and Audunson, R.: Use of library space and the library as place, Libr. Inform. Sci. Res., 34, 138-149, 2012.

Akalin, C., Baumann, A., Hartmann, B., Inhoffen, L., Kanthak, D., Nickels, M., and Wiedlich, W.: Schulden und Finanzen - Bonn muss sparen, General Anzeiger Bonn, 11. November 2012, online aufrufbar: http://www.general-anzeiger-bonn.de/bonn/stadt-bonn/ Schulden-und-Finanzen-Bonn-muss-sparen-article902204.html (letzter Zugriff: 4. August 2019), 2012.

ATZE Musiktheater GmbH: Offizielle Webseite des Festivals Dabba Dabba Du: Deutschlands größtem Festival für Kindermusik, online aufrufbar: http://www.dabbadabbadu.de/ konzerte-in-bibliotheken/, letzter Zugriff: 4. August 2019.

Audunson, R.: The public library as a meeting-place in a multicultural and digital context. The necessity of low-intensive meetingplaces, J. Doc., 61, 429-441, 2005.

Barlösius, E.: Der Anteil des Räumlichen an sozialer Ungleichheit und sozialer Integration: Infrastrukturen und Daseinsvorsorge, Sozialer Fortschritt, 58, 22-28, 2009.

Bernau, N.: Für die Metropolen-Bibliothek braucht es einen gewaltigen Neubau, Berliner Zeitung, 21. Januar 2020, online aufrufbar: https://www.berliner-zeitung.de/kulturvergnuegen/zlb-berlin-fuer-die-metropolen-bibliothek-brauchtes-einen-gewaltigen-neubau-li.5297, letzter Zugriff: 3. Februar 2020.

Bhabha, H. K.: The location of culture, Routledge, London, UK, New York, USA, 1994.

BIB e.V. (Berufsverband Information Bibliothek e.V.): Bibliothek 4.0 - wozu wir Dritte Orte brauchen!, Blogbeitrag vom 18. März 2019, online aufrufbar: https://b-u-b.de/ bibliothek-4-0-wozu-wir-dritte-orte-brauchen/, letzter Zugriff: 4. August 2019.

Birdi, B., Wilson, K., and Cocker, J.: The public library, exclusion and empathy: a literature review, Library Review, 57, 576-592, 2008.

Breher, N.: ZLB-Neubau könnte Vereinshaus zum Umzug zwingen, Tagesspiegel, 27. Januar 2020, online aufrufbar: https://www.tagesspiegel.de/berlin/zentrum-derdersim-kulturgemeinde-berlin-bedroht-zlb-neubau-koenntevereinshaus-zum-umzug-zwingen/25476780.html, letzter Zugriff: 3. Februar 2020

Brenner, N. and Theodore, N.: Cities and the geographies of ,actually existing neoliberalism“, Antipode, 34, 349-379, 2002.

Bundesstadt Bonn: Konzeptentwurf für die inhaltliche Zusammenarbeit von Volkshochschule und Stadtbibliothek einschließlich der Möglichkeit der Kinderbetreuung. Drucksache 1013971ED2, Eigenverlag der Bundesstadt Bonn, Bonn, Deutschland, 2010.

Bundesstadt Bonn: Kulturkonzept für die Stadt Bonn 2012-2022. Bonn, online aufrufbar: https://www.bonn.de/medien-global/ amt-41/Kulturkonzept_fuer_die_Stadt_Bonn_2012-2022_ Langfassung.pdf (letzter Zugriff: 4. August 2019), 2013.

Bundesstadt Bonn: Konzept für die Stadtbibliothek Bonn 2012 2022. Drucksache 1412893ED2, Eigenverlag der Bundesstadt Bonn, Bonn, Deutschland, 2014a.

Bundesstadt Bonn: Beschlussvorlage Zukünftige Bibliotheksstruktur. Drucksache 1412893, Eigenverlag der Bundesstadt Bonn, Bonn, Deutschland, 2014b. 
Bundesstadt Bonn: Bürgerantrag: Ablehnung Schließung Stadtteilbibliothek Bonn-Endenich. Drucksache: 1412826ED2, Eigenverlag der Bundesstadt Bonn, Bonn, Deutschland, 2014c.

Bundesstadt Bonn: Bürgerbeteiligung ,Bonn packt's an “ - Bürgervorschläge TOP 25 hier: Bürgervorschlag Rang-Nr.: 23 ,Kein Aus für Stadtteilbibliotheken, Grundversorgung in den Ortszentren statt Sahnehäubchen in Bonn-Innenstadt'. Drucksache 1510080, Eigenverlag der Bundesstadt Bonn, Bonn, Deutschland, 2014d.

Bundesstadt Bonn: Stadtbibliothek: Jahresbericht 2016, online aufrufbar: https://stadtbibliothekbonnblog.files.wordpress. com/2017/04/jahresbericht-2016.pdf (letzter Zugriff: 4. August 2019), 2017.

Bundesstadt Bonn: Stadtbibliothek: Jahresbericht 2017, online aufrufbar: https://stadtbibliothekbonnblog.files.wordpress. com/2018/06/stadtbibliothek-jahresbericht-2017_web.pdf (letzter Zugriff: 4. August 2019), 2018.

Bundesstadt Bonn: Bonn wächst. Aktuelle Zahlen aus dem Jahreswirtschaftsbe-richt 2018 zum Wachstum und zur Entwicklung der Bundesstadt Bonn, online aufrufbar: https: //www.bonn.de/themen-entdecken/wirtschaft-wissenschaft/ bonn-waechst.php, letzter Zugriff: 4. August 2019.

Carlsson, H.: Den nya stadens bibliotek: Om teknik, förnuft och känsla i gestaltningen av kunskapsoch upplevelsestadens folkbibliotek, PhD thesis, Lund University, Lund, Sweden, 2013.

Davies, J. S. and Blanco, I.: Austerity urbanism. Patterns of neoliberalisation and resistance in six cities of Spain and the UK, Environ. Plann. A, 49, 1517-1536, 2017.

dbv e.V. (Deutscher Bibliotheksverband e.V.): Bürgerschaftliches Engagement in Bibliotheken, online aufrufbar: https:// bibliotheksportal.de/informationen/beruf/ehrenamt/ (letzter Zugriff: 4. August 2019), 2014.

dbv e.V. (Deutscher Bibliotheksverband e.V.): Bericht zur Lage der Bibliotheken 2015, online aufrufbar: https://www.bibliotheksverband.de/fileadmin/user_upload/ DBV/publikationen/dbv_Bericht_zur_Lage_2015.pdf (letzter Zugriff: 4. August 2019), 2016.

dbv e.V. (Deutscher Bibliotheksverband e.V.): Bericht zur Lage der Bibliotheken 2017/18, online aufrufbar: https://www.bibliotheksverband.de/fileadmin/user_upload/ DBV/publikationen/dbv_Bericht_2017_Web.pdf (letzter Zugriff: 4. August 2019), 2018.

dbv e.V. (Deutscher Bibliotheksverband e.V.): Bericht zur Lage der Bibliotheken 2018/19, online aufrufbar: https://www.bibliotheksverband.de/fileadmin/user_upload/ DBV/publikationen/dbv_Jahresbericht2018_final.pdf, letzter Zugriff: 4. August 2019.

Di Marino, M. and Lapintie, K.: Libraries as transitory workspaces and spatial incubators, Libr. Inform. Sci. Res., 37, 118-129, 2015.

Donald, B., Glasmeier, A., Gray, M., and Lobao, L.: Austerity in the city: economic crisis and urban service decline?, Camb. J. Reg. Econ. Soc., 2014, 3-15, 2014.

Duveneck, A. and Volkholz, S.: Kommunale Bildungslandschaften, Herausgeber: Heinrich-Böll-Stiftung 2011. Band 9 der Reihe Bildung und Kultur, Berlin, Deutschland, 2011.

Esping-Andersen, G.: The Three Worlds of Welfare Capitalism, Princeton University Press, Princeton, USA, 1990.
Featherstone, D., Ince, A., MacKinnon, D., Strauss, K., and Cumbers, A.: Progressive localism and the construction of political alternatives, T. I. Brit. Geogr., 37, 177-182, 2012.

Flick, U.: Triangulation. Eine Einführung, Springer VS, Wiesbaden, Deutschland, 2004.

Freytag, T. and Hoyler, M.: Öffentliche und wissenschaftliche Bibliotheken, in: Nationalatlas Bundesrepublik Deutschland. Bildung und Kultur; Bd. 6, Herausgeber: Mayr, A. and Nutz, M., Institut für Länderkunde Leipzig, Spektrum, Heidelberg, Deutschland, 100-103, 2002.

Freytag, T. and Jahnke, H.: Perspektiven für eine konzeptionelle Orientierung der Bildungsgeographie, Geogr. Helv., 70, 75-88, https://doi.org/10.5194/gh-70-75-2015, 2015.

Gradmann, S. and Umlauf, K.: Die Bibliothek als Idee, in: Handbuch Bibliothek. Geschichte, Aufgaben, Perspektiven, Herausgeber: Umlauf, K. und Gradmann, S., J. B. Metzler, Stuttgart, Weimar, Deutschland, 3-32, 2012.

Graham, S. and Marvin, S.: Splintering Urbanism. Infrastrukturnetzwerke, technologische Mobilität und die Bedingung des Städtischen, in: Infrastrukturnetze und Raumentwicklung. Zwischen Universalisierung und Differenzierung, Herausgeber: Moss, T., Naumann, M., and Wissen, M., Oekom Verlag, München, Deutschland, 37-62, 2008.

Hastings, A., Bailey, N., Bramley, G., and Gannon, M.: Austerity urbanism in England: The 'regressive redistribution' of local government services and the impact on the poor and marginalized, Environ. Plann. A, 49, 2007-2024, 2017.

hbz (Hochschulbibliothekszentrum des Landes NordrheinWestfalen): Bibliotheken zählen! Berichtsjahr 2015: Auszüge aus der Deutschen Bibliotheksstatistik, online aufrufbar: https://www.hbz-nrw.de/service/mediathek/infografiken/ datenposter-bibliotheken-zaehlen-1 (letzter Zugriff: 4. August 2019), 2016.

Heinz, J.: Geplante Schließung der Stadtteilbibliothek Beuel-Ost: 1721 Unterschriften pro Bücherei, General Anzeiger Bonn, 23. Februar 2015, online aufrufbar: https://www.general-anzeiger-bonn.de/bonn/beuel/ 1721-unterschriften-pro-buecherei_aid-42252321 (letzter Zugriff: 4. August 2019), 2015.

Hobohm, H.-C.: Rechnen sich Bibliotheken? Vom Nutzen und Wert ihrer Leistungen, BuB Forum Bibliothek und Information, 59, 633-639, 2007.

Hobohm, H.-C.: Bibliothek im Wandel, in: Grundlagen der praktischen Information und Dokumentation, Herausgeber: Kuhlen, R., Semar, W., und Strauch, D., De Gruyter Saur, Berlin, Deutschland, 622-632, 2013.

Hobohm, H.-C.: Bibliothek und Diversität - eine theoretische Annäherung. Warum es eine zwingende Grundlegung für Bibliotheken ist, divers zu sein, arbido - Revue professionnelle pour archivistes, bibliothécaires et documentalistes 2017/1, Aarau \& Urtenen-Schönbühl, Schweiz, 2017.

Hvenegaard Rasmussen, C.: The participatory public library. The Nordic experience, New Library World, 117, 546-556, 2016.

Inhoffen, L.: Schließung von Stadtteilbibliotheken. Pro Stadtbezirk nur noch eine Bibliothek?, General Anzeiger Bonn, 16. Oktober 2014, online aufrufbar: http://www.general-anzeiger-bonn.de/bonn/stadt-bonn/ Pro-Stadtbezirk-nur-noch-eine-Bibliothek-article1475961.html (letzter Zugriff: 4. August 2019), 2014. 
Inhoffen, L.: 26,4 Millionen Euro-Projekt eingeweiht. Das Haus der Bildung ist eröffnet, General Anzeiger Bonn, 21. August 2015, online aufrufbar: https://www.general-anzeiger-bonn.de/bonn/ stadt-bonn/das-haus-der-bildung-ist-eroeffnet_aid-42481933 (letzter Zugriff: 4. August 2019), 2015.

Jochumsen, H., Hvenegaard Rasmussen, C., and Skot-Hansen, D.: The four spaces - a new model for the public library, New Library World, 113, 586-597, 2012.

Kearns, R.: Being There: Research through Observing and Participating, in: Qualitative research methods in human geography, Herausgeber: Hay, I., Oxford University Press, Oxford, UK, 103-121, 2000.

Knoche, M.: Die Idee der Bibliothek und ihre Zukunft, Wallstein Verlag, Göttingen, Deutschland, 2018.

Knopp, S.: Fortbestand ist garantiert. Vertrag zwischen Stadt und Stadtteilbibliotheken in Endenich, Dottendorf und Auerberg ist unterzeichnet, General Anzeiger Bonn, 21. Dezember 2015, 2015.

Kufner, A.: Eine Bibliothek, in der man sogar Kuchen backen kann, rbb24, 24. Oktober 2019, online aufrufbar: https://www.rbb24.de/kultur/beitrag/2019/10/ zentral-und-landesbibliothek-berlin-bibliothek-des-jahres.html (letzter Zugriff: 3. Februar 2020), 2019.

Lebuhn, H.: Das Neue Steuerungsmodell und die (Markt-)Logik städtischer Verwaltungen, Z - Zeitschrift Marxistische Erneuerung, 83, 35-45, 2010.

Libbe, J., Köhler, H., and Beckmann, K. J.: Infrastruktur und Stadtentwicklung. Technische und soziale Infrastrukturen - Herausforderungen und Handlungsoptionen für Infrastruktur- und Stadtplanung, Edition Difu - Stadt Forschung Praxis10, Berlin, Deutschland, 2010.

Mattert, J., Valentukeviciute, L., and Waßmuth, C.: Gemeinwohl als Zukunftsaufgabe: Öffentliche Infrastrukturen zwischen Daseinsvorsorge und Finanzmärkten, Schriften zu Wirtschaft und Soziales, Band 20. Heinrich-Böll-Stiftung in Zusammenarbeit mit Gemeingut in BürgerInnenhand (GiB) e.V., Berlin, Germany, online aufrufbar: https://www.gemeingut.org/wp-content/uploads/ 2017/06/Endf-Gemeinwohl-als-Zukunftsaufgabe_Web.pdf (letzter Zugriff: 4. August 2019), 2017.

Mayring, P. and Fenzl, T.: Qualitative Inhaltsanalyse, in: Handbuch Methoden der empirischen Sozialforschung, Zeitschrift Marxistische Erneuerung Baur, N. and Blasius, J., Springer VS, Wiesbaden, Deutschland, 633-648, 2019.

McFarlane, C. and Rutherford, J.: Political Infrastructures: Governing and Experiencing the Fabric of the City, Int. J. Urban Regional, 32, 363-374, 2008.

Michnik, K.: What threatens public libraries? The viewpoints of library directors in Sweden, New Library World, 115, 426-437, 2014.

Oldenburg, R.: The Great Good Place. Cafés, Coffee Shops, Bookstores, Bars, Hair Salons, and other Hangouts at the Heart Community, Paragon House, New York, USA, 1989.

Peck, J.: Austerity urbanism. American cities under extreme economy, City, 16, 626-655, 2012.

Peck, J.: Austerity Urbanism. The Neoliberal Crisis of American Cities. Rosa Luxemburg Stiftung. New York Office, online aufrufbar: http://www.rosalux-nyc.org/wp-content/files_mf/ peck_austerity_urbanism_eng93.pdf (letzter Zugriff: 4. August 2019), 2015.
Petzold, T. and Duveneck, A.: Austerity, (In)Justice and Space, Explorations in Space and Society, 47, 55-58, 2018.

Pinl, C.: Ehrenamt statt Sozialstaat? Kritik der Engagementpolitik, Aus Politik und Zeitgeschichte, 14/15, 49-54, 2015.

Pollio, A.: Technologies of austerity urbanism: the „smart city“ agenda in Italy (2011-2013), Urban Geogr., 37, 514-534, 2016.

Rada, U.: Bibliothek der Bestseller, taz, 7. Dezember 2014, online aufrufbar: https://taz.de/Mainstream-beim-Buecherkauf/ !5026740/ (letzter Zugriff: 3. Februar 2020), 2014.

Reidenbach, M., Bracher, T., Grabow, B., Schneider, S., and SeidelSchulze, A.: Investitionsrückstand und Investitionsbedarf der Kommunen, Edition Difu, Deutsches Institut für Urbanistik, Berlin, Deutschland, 2008.

Roeder, M.: Next Library ${ }^{\circledR}$ ? This Library!, online aufrufbar: https: //www.gemeingut.org/this-library/ (letzter Zugriff: 4. August 2019), 2018.

Sayah, A.: Architektur der neuen Bibliothek. Würfelspiele, innen und außen, Stuttgarter Zeitung, 21. Oktober 2011, online aufrufbar: https://www.stuttgarter-zeitung.de/inhalt.architektur-derneuen-bibliothek-wuerfelspiele-innen-und-aussen.b87785be97cd-4e6b-9bef-cc1608454b57.html (letzter Zugriff: 4. August 2019), 2011.

Schipper, S. and Schönig, B.: Impacts of the Global Financial Crisis on Cities in Europe. An Introduction to Urban Austerity, in: Urban Austerity. Impacts of the Global Financial Crisis on Cities in Europe, Herausgeber: Schipper, S. and Schönig, B., Theater der Zeit, Berlin, Deutschland, 7-19, 2016.

Seefeldt, J.: Öffentliche Bibliotheken und ihre Rolle für Bildung und Kultur in ländlichen Räumen, Kulturelle Bildung Online, online aufrufbar: https://www.kubi-online.de/artikel/oeffentlichebibliotheken-ihre-rolle-bildung-kultur-laendlichen-raeumen (letzter Zugriff: 29. Januar 2020), 2018.

Silomon-Pflug, F. and Heeg, S.: Neoliberale Neuordnung städtischer Verwaltungen am Beispiel des Liegenschaftsfonds Berlin, Geogr. Z., 101, 184-200, 2013.

Skot-Hansen, D., Hvenegaard Rasmussen, C., and Jochumsen, H.: The role of public libraries in culture-led urban regeneration, New Library World, 114, 7-19, 2013.

Soja, E. W.: Thirdspace: Journeys to Los Angeles and Other Realand-Imagined Places, Cambridge, Oxford, Blackwell, Oxford, UK, [1996] 2007.

Stadtbibliothek Bonn: Facebook-Auftritt der Stadtbibliothek Bonn, online aufrufbar: https://www.facebook.com/stadtbibliothek. bonn, letzter Zugriff: 4. August 2019.

Stadtbibliothek Chemnitz: E-Learning-Portal. Beitrag auf der offiziellen Homepage der Stadtbibliothek Chemnitz, online aufrufbar: http://www.stadtbibliothek-chemnitz.de/projekte-und-partner/ e-learning.html, letzter Zugriff: 4. August 2019.

Stadtbibliothek Köln: Blog der Stadtbibliothek Köln, online aufrufbar: https://stadtbibliothekkoeln.blog/2018/08/20/mint-festival/ (letzter Zugriff: 4. August 2019), 2018.

Stadtbibliothek Köln: Blog der Stadtbibliothek Köln, online aufrufbar: https://stadtbibliothekkoeln.blog/tag/makerspace/, letzter Zugriff: 4. August 2019.

Stadt Düsseldorf: LibraryLab, Beitrag auf der offiziellen Homepage der Stadt Düsseldorf, online aufrufbar: https://www.duesseldorf. de/stadtbuechereien/bibliotheken/librarylab.html, letzter Zugriff: 4. August 2019. 
Stollowsky, C. and Kurpjuweit, K.: Rechnungshof übt scharfe Kritik an ZLB-Plänen, Tagesspiegel, 3. Mai 2014, online aufrufbar: https://www.tagesspiegel.de/berlin/geplantelandesbibliothek-in-berlin-rechnungshof-uebt-scharfe-kritik-anzlb-plaenen/9840460.html (letzter Zugriff: 3. Februar 2020), 2014.

van Dyk, S. and Haubner, T.: Gemeinschaft als Ressource? Engagement und Freiwilligenarbeit im Strukturwandel des Wohlfahrtsstaats, in: Sozialstaat unter Zugzwang?, Herausgeber: Baumgartner, A. and Fux, B., Springer VS, Wiesbaden, Deutschland, 259279, 2019.

Vogt, H., Scheurer, B., and Pohla, H. B.: Der Makerspace: Ort für Kreativität und Wissenstransfer, BuB - Forum Bibliothek und Information, online aufrufbar: https://b-u-b.de/makerspace/ (letzter Zugriff: 4. August 2019), 2016.

Willcke, H.: Beide Bibliotheken sollen geöffnet bleiben. Büchereien: Beuel schickt ein starkes Signal nach Bonn, General Anzeiger Bonn, 19. März 2015, online aufrufbar: http://www.generalanzeiger-bonn.de/bonn/beuel/buechereien-Beuel-schickt-einstarkes-Signal-nach-Bonn-article1592912.html (letzter Zugriff: 4. August 2019), 2015.
Winkel, R.: Die Auswirkungen des demographischen Wandels auf die soziale Infrastruktur, in: Demographische Trends in Deutschland: Folgen für Städte und Regionen. Räumliche Konsequenzen des demographischen Wandels, Herausgeber: Gans, P. and Schmitz-Veltin, A., Forschungs- und Sitzungsberichte Akademie für Raumforschung und Landesplanung 226, Hannover, Deutschland, 172-191, 2006.

Wissen, M. and Naumann, M.: Raumdimensionen des Wandels technischer Infrastruktursysteme. Eine Einleitung, in: Infrastrukturnetze und Raumentwicklung. Zwischen Universalisierung und Differenzierung, Herausgeber: Moss, T., Naumann, M., and Wissen, M., Oekom Verlag, München, Deutschland, 17-34, 2008. 